\title{
Cholesterol in autism spectrum disorders
}

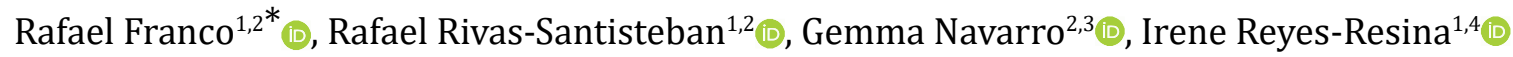 \\ ${ }^{1}$ Department Biochemistry and Molecular Biomedicine, School of Biology, University of Barcelona, 08028 Barcelona, Catalonia, \\ Spain \\ ${ }^{2}$ Centro de Investigación en Red, Enfermedades Neurodegenerativas (CiberNed), Instituto de Salud Carlos III, 28031 Madrid, \\ Madrid, Spain \\ ${ }^{3}$ Department of Biochemistry and Physiology, School of Pharmacy and Food Science, University of Barcelona, 08028 Barcelona, \\ Catalonia, Spain \\ ${ }^{4}$ Current address: RG Neuroplasticity, Leibniz Institute for Neurobiology, 39118 Magdeburg, Saxony-Anhalt, Germany
}

*Correspondence: Rafael Franco, Department Biochemistry and Molecular Biomedicine, School of Biology, University of Barcelona, Diagonal 643, 08028 Barcelona, Catalonia, Spain.rfranco@ub.edu; rfranco123@gmail.com

Academic Editor: Ta-Yuan Chang, Geisel School of Medicine at Dartmouth College, USA

Received: March 1, 2021 Accepted: May 7, 2021 Published: August 5, 2021

Cite this article: Franco R, Rivas-Santisteban R, Navarro G, Reyes-Resina I. Cholesterol in autism spectrum disorders. Explor Neuroprot Ther. 2021;1:10-8. https://doi.org/10.37349/ent.2021.00003

\begin{abstract}
The autism spectrum disorder (ASD) comprises a series of neurological diseases that share serious alterations of the development of the central nervous system. The degree of disability may vary so that Asperger's may have a relatively normal life and get positions of responsibility in corporations and even in Governments, whereas other ASD sufferers are fully dependent on caregivers and have serious cognitive deficits. Although the first cases of autism were detected by looking at failures in metabolism, e.g., phenylketonuria, to later identify the faulty gene, today the trend is the opposite, first obtaining the exome and minimizing the look for altered parameters in blood, urine, etc. Cholesterol is key for neural development as it is not able to cross the blood brain barrier. Therefore, any gene or environmental factor that affects cholesterol synthesis will impact early developmental stages eventually leading to a disease within the autism spectrum and/or schizophrenia. This review provides data of the relevance of cholesterol dyshomeostasis in autism spectrum disorders. Determining biochemical parameters in body fluids should help to provide new therapeutic approaches in some cases of autism.
\end{abstract}

\section{Keywords}

Autism spectrum disorder, Asperger syndrome, cholesterol, cholesterol dyshomeostasis, Rett syndrome, Smith-Lemli-Opitz syndrome, blood brain barrier

\section{Introduction}

Autism spectrum disorders (ASD) encompass any alteration in the development of the nervous system that leads to neurological/neuropsychiatric disturbances. Although Asperger's are considered within the cases of ASD, they constitute a more homogeneous class of patients and do not need special care when they reach

(C) The Author(s) 2021. This is an Open Access article licensed under a Creative Commons Attribution 4.0 International License (https://creativecommons.org/licenses/by/4.0/), which permits unrestricted use, sharing, adaptation, distribution and reproduction in any medium or format, for any purpose, even commercially, as long as you give appropriate credit to the original author(s) and the source, provide a link to the Creative Commons license, and indicate if changes were made. 
adulthood. In contrast, there are cases of ASD with severe neurological disorders, including self-injuries and requiring lifelong care.

Tests at birth include, for any child in developed countries, detection of some diseases that, in the absence of proper treatment, lead to neurodevelopmental defects. Two relevant examples are: defect in thyroxine production, which leads to cretinism coursing with mental retardation, and phenylketonuria due to mutations in an enzyme of the phenylalanine metabolism. Fortunately, these two diseases are quickly detected by means of blood/urine tests and efficacious treatments avoid neurological manifestations. The word autism was used to describe the patients with congenital phenylketonuria and, reciprocally, there are autism cases that course with phenylketonuria [1-6]. What these diseases share is abnormal nervous system development and alteration of metabolism, i.e. they are a kind of inheritable metabolic diseases. In this minireview we will focus on the alterations in cholesterol metabolism that are relatively frequent in ASD. On the one hand, the discovery of clues to emit hypothesis on which is the compromised step(s) in cholesterol metabolism may be helpful to stratify ASD cases. On the other hand, knowledge of the details leading to dysbalance may provide novel opportunities of therapy based on the use of supplements able to restore the homeostatic levels of cholesterol and related molecules. A topical review article considered some metabolic alterations that correlate with ASD cases and suggested that metabolic parameters in urine and blood should be done to, at the very least, be able to stratify some ASD patients [7].

\section{Cholesterol and biological membranes}

In mammals, cholesterol is necessary for the biosynthesis of many membranous components, including the plasma membrane. The higher content of lipids in the nervous system suggests that, among other lipids, cholesterol is key for physiological development of the nervous system. Actually, one of the membranes with higher cholesterol content is myelin, which is key for neurotransmission. The brain is the organ in the human body that requires more time to complete a full development. Therefore, any shortage of essential components compromises such development. Importantly the brain synthesizes its own cholesterol [8]. Therefore, the supply of the molecule is secured in the fetus until the blood-brain barrier (BBB) is established. When the $\mathrm{BBB}$ is functional cholesterol does not enter the brain, that depends on its own machinery to synthesize the compound. In summary, whereas cholesterol is usually in excess in the periphery due to de novo synthesis and consumption in food, the brain may have a shortage that will affect higher functions (Figure 1). The qualitative and quantitative consequences of cholesterol dyshomeostasis in terms of motor, cognition and further deficits, may depend on which is (are) the step(s) that are compromised.

FETAL DEVELOPMENT

ASD POST-NATAL

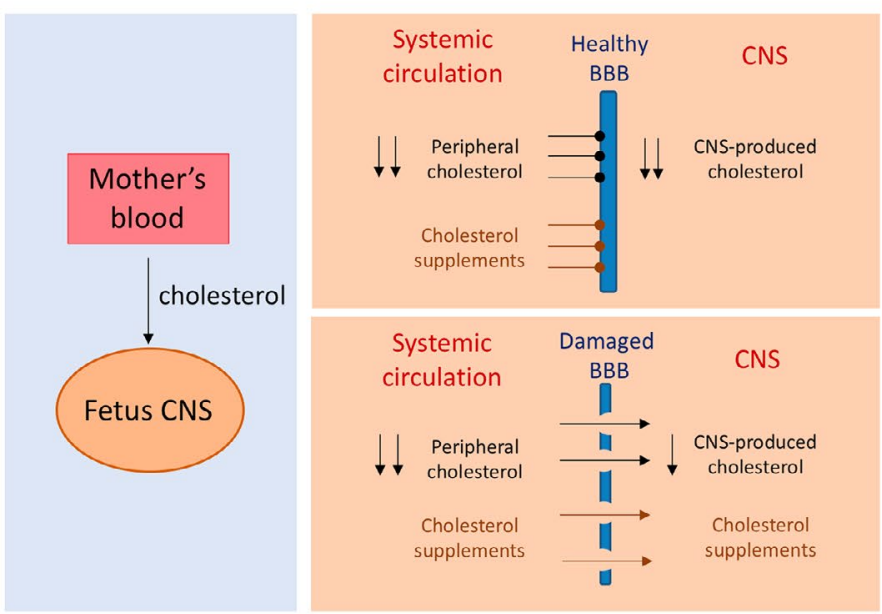

CHOLESTEROL AVAILABILITY vS SYMPTOMS

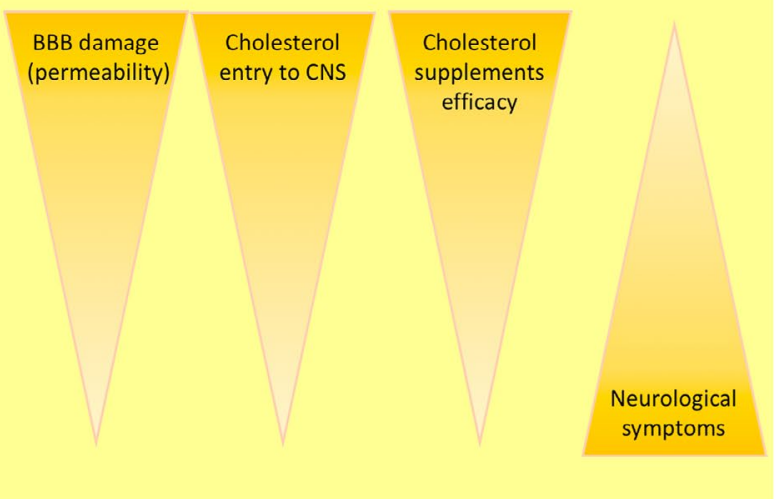

Figure 1. Flux of cholesterol in ASD coursing with cholesterol dyshomeostasis. During pregnancy (left), cholesterol from the mother reaches all organs in the fetus. After birth, cholesterol from the periphery of from supplements do not enter in the central nervous system (CNS) in the case of well-formed BBB but it could enter in a damaged BBB (center). In the latter case, the cholesterol from the supplements can enter the patient's CNS and improve neurological symptoms, since the features of ASD would depend on the availability of cholesterol (right) 


\section{The Smith-Lemli-Opitz syndrome}

The Smith-Lemli-Opitz syndrome is a rare disease caused by a defect in the synthesis of cholesterol. It is an autosomal recessive disorder that affects overall body homeostasis. Accordingly, malformations may occur in almost any organ and the kind of symptoms, from mild to severe, seemingly correlates with the residual potential of obtaining cholesterol and derivatives. Discovery of the altered gene product, 7-dehydrocholesterol reductase, in 1994 led to consider this syndrome the first true metabolic syndrome of multiple congenital malformations [9].

Mental retardation and neurological alterations are usually linked to microcephalia, hypoplasia and/ or absence of corpus callosum. The Diagnostic and Statistical Manual of Mental Disorders (now in its 5th edition: DSM- ${ }^{\circledR}$ ), which is an instrumental tool managed by the American Psychiatric Association, leads to estimates of ASD in more than $50 \%$ of cases and $86 \%$ if the "Pervasive Developmental Disorders, not otherwise specified" (PDD NOS) are counted [10]. As of 2006, this congenital disease was considered as the clearest example of single gene defect associated to autism. Interestingly not all patients respond equally to cholesterol supplements, something that led to suspect that, further to the gene alteration, there are variant autism types that support the variety of clinical casuistry within the autistic spectrum in this disease [10-13]. The blood parameters in these patients show clear alterations in cholesterol and sterol levels [10], thus pointing to cholesterol dyshomeostasis as the potential culprit in a significant number of children with ASD.

Clinical chemistry parameters were instrumental to detect cholesterol dyshomeostasis in ASD cases not related to Smith-Lemli-Opitz syndrome. As this syndrome can be diagnosed by blood tests, Tierney and colleagues [11] noticed that in 17 out of 100 ASD cases the level of blood cholesterol was very low $(<100$ $\mathrm{mg} / 100 \mathrm{~mL}$ ) despite they did not have the Smith-Lemli-Opitz syndrome. Blood was obtained from the Autism Genetic Resource Exchange (AGRE) sample repository filtering to only include families with 2 or more ASD cases. Therefore, shortage of cholesterol is related to some cases of ASD. In summary of 100 ASD cases not linked to the congenital disease, 17 had deficits in blood cholesterol levels. The other cases had levels > $100 \mathrm{mg} / 100 \mathrm{~mL}$; it should be noted that cholesterol in this study was measured by gas-chromatography, i.e. samples were not quantitated according to the cholesterol in lipoprotein approach. As the authors point out, one limitation of the study is the lack of information about lipoprotein profiles.

\section{Rett syndrome}

The Rett syndrome (OMIM 312750) is a neurodevelopmental disorder associated to genetic abnormalities in the X chromosome that affects one in 10,000-15,000 females. Most of the cases are associated with mutations in the gene for methyl-CpG-binding protein 2 (MECP2), a transcription factor. To our knowledge there was about a decade ago that clear evidence of cholesterol metabolism alteration occurred in patients. Genotyping and sequencing methods led to discover squalene epoxidase as one gene that could rescue the deficits observed in MECP2 knockout mice. As the enzyme is important for cholesterol synthesis, it was further shown that MECP2 knockout mouse males displayed clear signs of altered cholesterol metabolism [14, 15]. Taking this study as reference, Segatto et al. [16] reported one year later that total cholesterol and low density lipoproteins (LDL) were elevated in the serum of Rett's patients. The finding was confirmed within the framework of the Rett search consortium study [17] that considers of metabolic origin the symptoms of the disease [15]. One of the most important enzymes of cholesterol synthetic pathway, 3-hydroxy-3-methyl glutaryl coenzyme A reductase was increased in skin fibroblasts from patients. In addition, fibroblasts of patients showed altered levels of various proteins related to cholesterol homeostasis and/or lipoprotein handling, e.g., sterol regulatory element binding proteins, LDL receptor or scavenger receptor B-1 $[16,18]$. Taken together these results are intriguing: unlike in the Smith-Lemli-Opitz syndrome, the Rett syndrome courses with elevations of plasma cholesterol. As the alteration of cholesterol metabolism occurs in both syndromes it is tempting to speculate that the issues related to mental retardation and/or cognitive/motor deficits are not due to abnormal cholesterol 
production and/or handling in the periphery but in the CNS; a collection of facts and hints supporting this hypothesis is provided elsewhere $[19,20]$.

\section{Further evidence of cholesterol links to ASD cases}

A full account of all evidence linking some autism cases to cholesterol dyshomeostasis is out of the scope of the present mini-review. Accordingly, only few examples will be here presented. One of them derives from the serendipitous discovery that ganglioside GM1 affects the distribution of cholesterol in cell membranes. Biophysical studies showed that in the absence of the ganglioside distribution of cholesterol in biomimetic lipid bilayers is symmetric. In contrast, addition of GM1 leads to an asymmetric distribution of GM1 and cholesterol that suggest that the two molecules interact in biological membranes [21]. Remarkably, the erythrocyte membrane of patients of 16 ASD cases showed abnormal GM1/cholesterol ratio. Age ranged from 3 to 8 with grade severity between 30 and 53. Red blood cells in ASD had less cholesterol and more GM1 than in erythrocytes from age (3 to 8-year-old)-matched controls. Authors pointed out a "need for further investigation of lipid metabolism in autistic subjects" [22].

An ingenious study aimed to correlate ASD in the offspring of mothers with dyslipidemia. In addition to cholesterol and lipoproteins, the level of branched-chain amino acids (leucine, isoleucine, and valine) was post-partum determined. Results in 756 mother-son/daughter pairs from the Boston birth cohort underscored a high risk of ASD (86 cases in total) in sons of mothers with high-density lipoprotein cholesterol (HDLc) and branched-chain amino acids above of the average [23]. Although the underlying mechanisms are not yet known, the robustness of the results in such a small cohort indicates that metabolic alterations at the gestational level may impact the risk of ASD. It is suggested that the level of amino acids should be simultaneously analyzed in the body fluids (e.g., in saliva) of ASD patients and the data standardized according to gender, age, intelligence quotient and psychoactive medication when the assay is performed [24]. On the one hand, branched-chain amino acids are necessary for protein synthesis, but they are also important to regulate, in certain situations and/or under certain diets, plasma glucose levels. On the other hand, mutations in genes coding for enzymes that handle those compounds, e.g., branched-chain ketoacid dehydrogenase may lead to neurological manifestations $[25,26]$. Very intriguing is the fact that the correlation was higher in sons than in daughters [23]. Also intriguing is the correlation between ASD risk in progeny and low LDL levels in obese mothers, but not in mothers of normal weight [27].

Due to the huge variety of possible causes and symptoms within the autistic spectrum, it is not easy to develop animal models of the disease. One of them consists of prenatal exposure of rats to valproate, an anti-epileptic drug. The cholesterol/isoprenoid metabolism was analyzed in animals in uterus exposed to the drug. Cholesterol/isoprenoid dyshomeostasis was found in various brain areas of those animals at different ages, from young and "adolescent" to adult rats. The animals also displayed a reduced number of oligodendrocytes with signs of demyelination in the hippocampus [28]. A phenotypic model, which is commercially available through Charles River (Wilmington, MA, USA), is the Black and Tan BRachyury $\mathrm{T}+\mathrm{Itpr} 3 \mathrm{tf} / \mathrm{J}$ (BTBR $\mathrm{T}+\mathrm{tf} / \mathrm{J}$ ) mouse. It is an inbred line that already in juvenile states shows phenotypes comparable to those necessary for the diagnosis of autism, that is, stereotyped patterns, reduced playing time and aberrant social interactions [29-32]. The brain of those animals has gross anatomical abnormalities if compared with the C57Bl/6J mouse [33]. A genetic model is the Fragile X Mental Retardation 1 knockout (Fmr1-KO) mouse that, apart from autism-like behavior [34], displays altered dopaminergic and $\gamma$-amino butyric acid (GABA)-ergic pathways [35, 36]. There are further models, among other, the mutant SH3 and multiple ankyrin repeat domains 3 (Shank) mice [37] or the transgenic mice expressing different copy number of gene for Calcium-Dependent Activator Protein For Secretion 2 (Cadps2) [38-40]; they are very valuable despite they cannot encompass the variety of etiologies of ASD.

Recently, the new approach known as "multimodal precision medicine" has given a boost to the hypothesis that some cases of ASD are related in one way or another to cholesterol dyshomeostasis. The approach is very complex and the study by Luo et al. [41] includes, among other, health records, information from whole exome sequencing within members of families, expression of proteins along human development 
using 524 brain samples from 42 subjects, clustering of exons co-expressed during early development and health records and identification of ASD-segregating deleterious variations. The study also considered the approximate 4:1 male/female ratio in ASD. As the authors pointed out, their analysis "identified 33 neurodevelopmentally co-regulated, sex-differentially expressed clusters with ASD-segregating deleterious variation" [41]. In what concerns alterations of cholesterol levels and/or of cholesterol metabolism, the study identifies a subgroup of patients with dyslipidemia-associated autism. On looking at the clinical chemistry data of $>2.7$ million individuals and > 25,000 ASD children seen at Harvard Medical Children's Hospital (Boston, USA), patients displayed lipid profiles that were out of the physiological range. In addition, mining databases containing parameters from mice having targeted deletions of genes involved in lipidemia or in ASD, revealed that some ASD models "are more similar to dyslipidemia mice than to other ASD models" [41], meaning that some ASD models have lipid dyshomeostasis and some mice models of dyslipidemia display social and cognitive abnormalities.

\section{Is it possible to supply cholesterol to the brain of ASD patients?}

Children with Smith-Lemli-Opitz syndrome improve after cholesterol supplements. Not only many of the symptoms due to malformations in peripheral organs are improved but the compound also decreased the level of anxiety, temper outbursts, aggressiveness, self-injury, etc. [12]. These results are important because they question the hypothesis that the CNS synthesizes its own cholesterol. Either there is a portion of cholesterol that may cross the BBB or the BBB of these patients is permeable to cholesterol, perhaps by malformation (See Figure 1). Unfortunately, there are no definitive data to show that the BBB in Smith-LemliOpitz syndrome is as functional as in healthy individuals.

Even considering that BBB is not permeable to cholesterol, a congenital defect in the main enzyme responsible for its synthesis can be improved by supplementation with cholesterol, as this would lead to the synthesis of vitamin D, synthesis of sex hormones, synthesis of steroid hormones, etc. Normalization of the level of these hormones would improve the general well-being of the patient. However, in ASD coursing with cholesterol dyshomeostasis the main challenge is to be able to deliver the compound to the CNS in the amounts needed for appropriate nervous system development.

CNS cholesterol levels in ASD may vary depending on many factors. One of them is the amount of enzyme activity that remains in the case, for instance, of altered genes leading to mutant enzymes or of altered levels of transcription factors that in turn leads to lower levels of non-mutated enzyme. Enzyme activators are difficult to develop and probably the best option is to rely in the new technologies of target delivery [42, 43]. We think that after careful assessment of safety, a clinical trial using cholesterol-containing nanocarriers targeting the CNS could be undertaken in ASD cases coursing with cholesterol dyshomeostasis. Controlled by ClinicalTrials.org, there are up to 465 studies using nanoparticles. However, the use of this technique in CNS pathologies lags behind others due to the difficulty of addressing whether or not the carrier and its content reach the proper target. We have identified one designed to combat recurrent glioblastomas using a plasmid encapsulated in a liposome. Phase II is now complete, but the number of participants was very low and promoters decided to terminate it (Ref. \#NCT02340156).

Gene therapy using adenoviruses, which have been recently introduced in human therapy in the form of vaccines to combat COVID-19 [44] and are tested in animal models for combating CNS diseases [45, 46], could be useful but only in cases in which the faulty gene is identified, e.g., in the case of MECP2 whose mutations are associated to the Rett syndrome (see above).

The use of precursors of cholesterol is an option that might be explored. Mevalonic acid, the product of the first step of cholesterol synthesis, is present in blood and can be administered as a supplement $[47,48]$. We have been unable to find reliable data on the possibility that mevalonate crosses the BBB, therefore it is suggested that this possibility may be explored in experimental animals. Provided mevalonic acid crosses the BBB, it would be of interest to see whether supplements of this compound could alleviate symptoms in ASD cases related to dyslipidemia. The only concern is that excess peripheral mevalonate may lead to substantial 
increases in blood cholesterol levels. However, this side effect could be easily addressed by using statins unable to cross the BBB.

\section{Conclusions}

On the one hand, stratification in ASD is an urgent need. On the other hand, cumulative evidence supports the existence of dyslipidemia-associated ASD. Necessary when there is suspicion of cholesterol dyshomeostasis in ASD is an ad hoc blood test where branched-chain amino acids, triglycerides, and HDL/LDL cholesterol and steroid hormones, are determined. Also important is to know the blood levels of precursors of cholesterol such as mevalonic acid. Such blood biochemical parameters would serve to better know the causes of symptoms and to refine the therapeutic management of the patient. The sooner the dyslipidemia is identified, the better the prospects for early interventions. Hopefully, the future will tell how to improve the delivery to the brain of lipids needed for physiological or near physiological nervous system development.

\section{Abbreviations}

ASD: autism spectrum disorder

BBB: blood-brain barrier

CNS: central nervous system

LDL: low-density lipoprotein

MECP2: methyl-CpG-binding protein 2

\section{Declarations}

\section{Acknowledgements}

In memoriam, Geoffrey Burnstock (May 1929-June 2020) was the discoverer of purinergic nerves, the best pharmacologist Rafael Franco has ever met and a multitalented and inspiring human being. María Teresa Miras-Portugal (February 1948-May 2021), a leading scientist in the field of purinergic research and a very dear friend of Rafael Franco.

\section{Author contributions}

RF and IRR designed the review. All authors participated in literature search and selection of suitable papers. $\mathrm{RF}$ wrote the first draft. IRR prepared the figure. All authors edited the paper and approved the final version.

\section{Conflicts of interest}

The authors declare that they have no conflicts of interest.

\section{Ethical approval}

Not applicable.

\section{Consent to participate}

Not applicable.

\section{Consent to publication}

Not applicable.

\section{Availability of data and materials}

Not applicable. 


\section{Funding}

This work was partially supported by grants SAF2017-84117, RTI2018-098830-B-I00, and PID2019109240RB-I00 from the Spanish Ministry of Science and Innovation (MICINN) and or Science, Innovation and Universities; they may include EU FEDER funds. The laboratory of the University of Barcelona is considered of excellence by the Regional Catalonian Government (grup consolidat \#2017 SGR 1497), which does not provide any specific funding for personnel, equipment and reagents or for payment of services.

\section{Copyright}

(C) The Author(s) 2021.

\section{References}

1. Driscoll KW, Hsia DY. Detection of the heterozygous carriers of phenylketonuria. Lancet. 1956;271:1337-8.

2. Jervis GA. Detection of heterozygotes for phenylketonuria. Clin Chim Acta. 1960;5:471-6.

3. Cawte JE. Phenylketonuria. Med J Aust. 1954;2:15-9.

4. Baieli S, Pavone L, Meli C, Fiumara A, Coleman M. Autism and phenylketonuria. J Autism Dev Disord. 2003;33:201-4.

5. Lowe TL, Tanaka K, Seashore MR, Young JG, Cohen DJ. Detection of phenylketonuria in autistic and psychotic children. JAMA. 1980;243:126-8.

6. Friedman E. The "autistic syndrome" and phenylketonuria. Schizophrenia. 1969;1:249-61.

7. Manzi B, Loizzo AL, Giana G, Curatolo P. Autism and metabolic diseases. J Child Neurol. 2008;23:307-14.

8. Björkhem I, Meaney S. Brain cholesterol: long secret life behind a barrier. Arterioscler Thromb Vasc Biol. 2004;24:806-15.

9. Tint GS, Irons M, Elias ER, Batta AK, Frieden R, Chen TS, et al. Defective cholesterol biosynthesis associated with the Smith-Lemli-Opitz syndrome. N Engl J Med. 1994;330:107-13.

10. Sikora DM, Pettit-Kekel K, Penfield J, Merkens LS, Steiner RD. The near universal presence of autism spectrum disorders in children with Smith-Lemli-Opitz syndrome. Am J Med Genet A. 2006;140:1511-8.

11. Tierney E, Bukelis I, Thompson RE, Ahmed K, Aneja A, Kratz L, et al. Abnormalities of cholesterol metabolism in autism spectrum disorders. Am J Med Genet B Neuropsychiatr Genet. 2006;141B:666-8.

12. Aneja A, Tierney E. Autism: the role of cholesterol in treatment. Int Rev Psychiatry. 2008;20:165-70.

13. Opitz JM, Penchaszadeh VB, Holt MC, Spano LM. Smith-Lemli-Opitz (RSH) syndrome bibliography. Am J Med Genet. 1987;28:745-50.

14. Buchovecky CM, Turley SD, Brown HM, Kyle SM, McDonald JG, Liu B, et al. A suppressor screen in Mecp2 mutant mice implicates cholesterol metabolism in Rett syndrome. Nat Genet. 2013;45:1013-20.

15. Justice MJ, Buchovecky CM, Kyle SM, Djukic A. A role for metabolism in Rett syndrome pathogenesis: new clinical findings and potential treatment targets. Rare Dis. 2013;1:e27265.

16. Segatto M, Trapani L, Di Tunno I, Sticozzi C, Valacchi G, Hayek J, et al. Cholesterol metabolism is altered in Rett syndrome: a study on plasma and primary cultured fibroblasts derived from patients. PLoS One. 2014;9:e104834.

17. Neul JL, Kaufmann WE, Glaze DG, Christodoulou J, Clarke AJ, Bahi-Buisson N, et al; RettSearch Consortium. Rett syndrome: revised diagnostic criteria and nomenclature. Ann Neurol. 2010;68:944-50.

18. Sticozzi C, Belmonte G, Pecorelli A, Cervellati F, Leoncini S, Signorini C, et al. Scavenger receptor B1 posttranslational modifications in Rett syndrome. FEBS Lett. 2013;587:2199-204.

19. Segatto M, Tonini C, Pfrieger FW, Trezza V, Pallottini V. Loss of mevalonate/cholesterol homeostasis in the brain: a focus on autism spectrum disorder and Rett syndrome. Int J Mol Sci. 2019;20:3317. 
20. Gillberg C, Fernell E, Kočovská E, Minnis H, Bourgeron T, Thompson L, et al. The role of cholesterol metabolism and various steroid abnormalities in autism spectrum disorders: a hypothesis paper. Autism Res. 2017;10:1022-44.

21. Rondelli V, Fragneto G, Motta S, Del Favero E, Brocca P, Sonnino S, et al. Ganglioside GM1 forces the redistribution of cholesterol in a biomimetic membrane. Biochim Biophys Acta. 2012;1818:2860-7.

22. Schengrund CL, Ali-Rahmani F, Ramer JC. Cholesterol, GM1, and autism. Neurochem Res. 2012;37:1201-7.

23. Panjwani AA, Ji Y, Fahey JW, Palmer A, Wang G, Hong X, et al. Maternal dyslipidemia, plasma branchedchain amino acids, and the risk of child autism spectrum disorder: evidence of sex difference. J Autism Dev Disord. 2020;50:540-50.

24. Zheng HF, Wang WQ, Li XM, Rauw G, Baker GB. Body fluid levels of neuroactive amino acids in autism spectrum disorders: a review of the literature. Amino Acids. 2017;49:57-65.

25. Burrage LC, Nagamani SCS, Campeau PM, Lee BH. Branched-chain amino acid metabolism: from rare Mendelian diseases to more common disorders. Hum Mol Genet. 2014;23:R1-8.

26. Strauss KA, Carson VJ, Soltys K, Young ME, Bowser LE, Puffenberger EG, et al. Branched-chain $\alpha$-ketoacid dehydrogenase deficiency (maple syrup urine disease): treatment, biomarkers, and outcomes. Mol Genet Metab. 2020;129:193-206.

27. Park BY, Yao R, Tierney E, Brucato M, Hong X, Wang G, et al. The association between maternal lipid profile after birth and offspring risk of autism spectrum disorder. Ann Epidemiol. 2021;53:50-5.e1.

28. Cartocci V, Catallo M, Tempestilli M, Segatto M, Pfrieger FW, Bronzuoli MR, et al. Altered brain cholesterol/ isoprenoid metabolism in a rat model of autism spectrum disorders. Neuroscience. 2018;372:27-37.

29. Llaneza DC, DeLuke SV, Batista M, Crawley JN, Christodulu KV, Frye CA. Communication, interventions, and scientific advances in autism: a commentary. Physiol Behav. 2010;100:268-76.

30. Moy SS, Nadler JJ, Perez A, Barbaro RP, Johns JM, Magnuson TR, et al. Sociability and preference for social novelty in five inbred strains: an approach to assess autistic-like behavior in mice. Genes Brain Behav. 2004;3:287-302.

31. Moy SS, Nadler JJ, Young NB, Perez A, Holloway LP, Barbaro RP, et al. Mouse behavioral tasks relevant to autism: phenotypes of 10 inbred strains. Behav Brain Res. 2007;176:4-20.

32. McFarlane HG, Kusek GK, Yang M, Phoenix JL, Bolivar VJ, Crawley JN. Autism-like behavioral phenotypes in BTBR T+tf/J mice. Genes Brain Behav. 2008;7:152-63.

33. Mercier F, Cho Kwon Y, Kodama R. Meningeal/vascular alterations and loss of extracellular matrix in the neurogenic zone of adult BTBR T +tf/J mice, animal model for autism. Neurosci Lett. 2011;498:173-8.

34. Oddi D, Crusio WE, D'Amato FR, Pietropaolo S. Monogenic mouse models of social dysfunction: implications for autism. Behav Brain Res. 2013;251:75-84.

35. Chao OY, Pathak SS, Zhang H, Dunaway N, Li JS, Mattern C, et al. Altered dopaminergic pathways and therapeutic effects of intranasal dopamine in two distinct mouse models of autism. Mol Brain. 2020;13:111.

36. Paluszkiewicz SM, Martin BS, Huntsman MM. Fragile X syndrome: the GABAergic system and circuit dysfunction. Dev Neurosci. 2011;33:349-64.

37. Yoo J, Bakes J, Bradley C, Collingridge GL, Kaang BK. Shank mutant mice as an animal model of autism. Philos Trans R Soc Lond B Biol Sci. 2013;369:20130143.

38. Sadakata T, Shinoda Y, Sato A, Iguchi H, Ishii C, Matsuo M, et al. Mouse models of mutations and variations in autism spectrum disorder-associated genes: mice expressing Caps2/Cadps2 copy number and alternative splicing variants. Int J Environ Res Public Health. 2013;10:6335-53. 
39. Sadakata T, Furuichi T. Developmentally regulated $\mathrm{Ca}^{2+}$-dependent activator protein for secretion 2 (CAPS2) is involved in BDNF secretion and is associated with autism susceptibility. Cerebellum. 2009;8:312-22.

40. Sadakata T, Furuichi T. $\mathrm{Ca}^{2+}$-dependent activator protein for secretion 2 and autistic-like phenotypes. Neurosci Res. 2010;67:197-202.

41. Luo Y, Eran A, Palmer N, Avillach P, Levy-Moonshine A, Szolovits P, et al. A multidimensional precision medicine approach identifies an autism subtype characterized by dyslipidemia. Nat Med. 2020;26:1375-9.

42. Zhou Z, Sun T, Jiang C. Recent advances on drug delivery nanocarriers for cerebral disorders. Biomed Mater. 2021;16:024104.

43. Cintra e Silva DDO, Estevanato LLC, Simioni AR, de Andrade Rodrigues MM, Lacava BM, Lacava ZGM, et al. Successful strategy for targeting the central nervous system using magnetic albumin nanospheres. J Biomed Nanotechnol. 2012;8:182-9.

44. Chung JY, Thone MN, Kwon YJ. COVID-19 vaccines: the status and perspectives in delivery points of view. Adv Drug Deliv Rev. 2021;170:1-25.

45. Li Y, Miller CA, Shea LK, Jiang X, Guzman MA, Chandler RJ, et al. Enhanced efficacy and increased long-term toxicity of CNS-directed, AAV-based combination therapy for Krabbe disease. Mol Ther. 2021;29:691-701.

46. Pickering CA, Mazarakis ND. Viral vector delivery of DREADDs for CNS therapy. Curr Gene Ther. 2021; [Epub ahead of print].

47. Jiao X, Ashtari N, Rahimi-Balaei M, Chen QM, Badbezanchi I, Shojaei S, et al. Mevalonate cascade and neurodevelopmental and neurodegenerative diseases: future targets for therapeutic application. Curr Mol Pharmacol. 2017;10:115-40.

48. Tonini C, Segatto M, Pallottini V. Impact of sex and age on the mevalonate pathway in the brain: a focus on effects induced by maternal exposure to exogenous compounds. Metabolites. 2020;10:304. 